\title{
Influence of torsion on the inelastic response of three-dimensional r.c. frames
}

\begin{abstract}
A three-dimensional reinforced concrete framed building was modelled using finite element method. Two types of elements, the beam-column element and flat shell element were used for modelling the frame and floor slabs, respectively. A computer program has been developed for the analysis of 3D framed building by integrating the finite element and stiffness method. The lumped inelasticity model with three-dimensional point hinges at the ends of the beam-column element was implemented. A yield surface for a reinforced section of the member subjected to simultaneous actions of biaxial bending, torsion and axial forces was evolved. The developed yield surface integrated with the theory of plasticity was used to develop a suitable procedure for inelastic analysis of three-dimensional problems with the floor slab assumed to remain elastic throughout the analysis. The inelastic procedure is able to predict the sequential formation of plastic hinges in the frame members and the continuous deterioration of the stiffness of the frame. A single storey one bay reinforced concrete space frame was analysed for twist loading to study the inelastic response of the reinforced concrete frame. The results indicate that, the consideration of torsion in defining the yielding surface plays a significant role in the inelastic behaviour and estimation of failure load for reinforced concrete frames under torsional loading.
\end{abstract}

Keyword: Torsion, 3D frame, Inelastic analysis, Finite element method, Yield surface 